\title{
RNA sequencing reveals an additional Crz1- binding motif in promoters of its target genes in the human fungal pathogen Candida albicans
}

\author{
Huihui Xu1, Tianshu Fang ${ }^{1}$, Raha Parvizi Omran², Malcolm Whiteway ${ }^{2}$ and Linghuo Jiang ${ }^{1^{*}}$ (1)
}

\begin{abstract}
Background: The calcium/calcineurin signaling pathway is mediated by the transcription factors NFAT (nuclear factor of activated T cells) in mammals and Crzl (calcineurin-responsive zinc finger 1) in yeasts and other lower eukaryotes. A previous microarray analysis identified a putative Crzl-binding motif in promoters of its target genes in Candida albicans, but it has not been experimentally demonstrated.

Methods: An inactivation mutant for CaCRZ1 was generated through CRISPR/Cas9 approach. Transcript profiling was carried out by RNA sequencing of the wild type and the inactivation mutant for CaCRZ1 in response to $0.2 \mathrm{M}$ $\mathrm{CaCl}_{2}$. Gene promoters were scanned by the online MEME (Multiple Em for Motif Elicitation) software. Gel electrophoretic mobility shift assay (EMSA) and chromatin immunoprecipitation (ChIP) analysis were used for in vitro and in vivo CaCrz1-binding experiments, respectively.

Results: RNA sequencing reveals that expression of 219 genes is positively, and expression of 59 genes is negatively, controlled by $\mathrm{CaCrz1}$ in response to calcium stress. These genes function in metabolism, cell cycling, protein fate, cellular transport, signal transduction, transcription, and cell wall biogenesis. Forty of these positively regulated 219 genes have previously been identified by DNA microarray analysis. Promoter analysis of these common 40 genes reveals a consensus motif [5'-GGAGGC(G/A)C(T/A)G-3'], which is different from the putative CaCrz1-binding motif [5'$\mathrm{G}(\mathrm{C} / \mathrm{T}) \mathrm{GGT}-3^{\prime}$ ] identified in the previous study, but similar to Saccharomyces cerevisiae ScCrz1-binding motif [5'-GNGG $\mathrm{C}(\mathrm{G} / \mathrm{T}) \mathrm{CA}-\mathrm{3}^{\prime}$. EMSA and ChIP assays indicate that $\mathrm{CaCrz}$ binds in vitro and in vivo to both motifs in the promoter of its target gene CaUTR2. Promoter mutagenesis demonstrates that these two CaCrz1-binding motifs play additive roles in the regulation of CaUTR2 expression. In addition, the CaCRZ1 gene is positively regulated by CaCrz1. CaCrz1 can bind in vitro and in vivo to its own promoter, suggesting an autoregulatory mechanism for CaCRZ1 expression.
\end{abstract}

Conclusions: $\mathrm{CaCrz} 1$ differentially binds to promoters of its target genes to regulate their expression in response to calcium stress. CaCrz1 also regulates its own expression through the 5'-TGAGGGACTG-3' site in its promoter.

Keywords: Candida albicans, Crz1, Calcium signaling, Transcription profiling, RNA sequencing, Promoter

\footnotetext{
* Correspondence: linghuojiang@sdut.edu.cn

${ }^{1}$ Laboratory for Yeast Molecular and Cell Biology, Department of Food

Science, School of Agricultural Engineering and Food Science, Shandong

University of Technology, Zibo 255000, China

Full list of author information is available at the end of the article
}

(c) The Author(s). 2019 Open Access This article is distributed under the terms of the Creative Commons Attribution 4.0 International License (http://creativecommons.org/licenses/by/4.0/), which permits unrestricted use, distribution, and

reproduction in any medium, provided you give appropriate credit to the original author(s) and the source, provide a link to the Creative Commons license, and indicate if changes were made. The Creative Commons Public Domain Dedication waiver (http://creativecommons.org/publicdomain/zero/1.0/) applies to the data made available in this article, unless otherwise stated. 


\section{Plain English summary}

Calcium ions regulate many cellular processes in both prokaryotes and eukaryotes, from bacteria to humans. Regulation of intracellular calcium homeostasis is highly conserved in eukaryotic cells. Gene expression in response to calcium stress is controlled by the calcium/calcineurin signalling through the transcription factors NFAT (the nuclear factor of activated T cells) in mammals and Crz1 (calcineurin-responsive zinc finger 1) in yeasts and other lower eukaryotes. Extracellular calcium stress causes an increase in cytosolic calcium, which leads to the binding of calcium ions to calmodulin that triggers activation of the protein phosphatase, calcineurin. Activated calcineurin dephosphorylates Crz1 in the cytosol, which leads to nuclear localization of Crz1 and its binding to promoters of its target genes to regulate their expression. Candida albicans is one of the most important human yeast pathogens. A previous microarray analysis identified a putative CaCrz1binding motif in promoters of its target genes in C. albicans, but it has not been experimentally demonstrated. Using a new technology, RNA sequencing, we have identified 219 genes that are positively, and 59 genes that are negatively, controlled by $\mathrm{CaCrz1}$ in response to calcium stress in this study. We have also revealed and demonstrated experimentally a novel consensus CaCrz1-binding motif [5'-GGAGGC(G/A)C(T/A)G-3'] in promoters of its target genes. In addition, we have discovered that CaCrz1 can bind to its own promoter, suggesting an autoregulatory mechanism for CaCRZ1 expression. These findings would contribute to our further understanding of molecular mechanisms regulating calcium homeostasis.

\section{Backgound}

Calcium ions regulate many cellular processes in both prokaryotes and eukaryotes, from bacteria to humans [1-5]. Intracellular calcium homeostasis is maintained by calcium transporters and sequestrators in the plasma and organelle membranes in eukaryotes. Regulation of calcium homeostasis is highly conserved in eukaryotic cells. Gene expression in response to calcium stress is controlled by the calcium/ calcineurin signalling through the transcription factor Crz1 in fungi or the nuclear factor of activated T cells (NFAT) in mammals [6, 7]. In Saccharomyces cerevisiae, an increase in cytosolic calcium triggers the calmodulin $/ \mathrm{Ca}^{2+}$ binding and activation of the protein phosphatase, calcineurin. Activated calcineurin dephosphorylates ScCrz1 in the cytosol, which leads to nuclear localization of ScCrz1 and its binding to promoters of its target genes, including the vacuolar calcium pump gene ScPMC1, the ER/Golgi calcium pump gene $S c P M R 1$ and the $S c R C H 1$ gene encoding the negative regulator of calcium uptake in the plasma membrane [810]. A genome-scale genetic screen has revealed additional genes that are involved in the regulation of calcium homeostasis in budding yeast [11].
Candida albicans remains as one of leading human fungal pathogens in immunocompromised patients [1214]. Functional counterparts of calcium homeostasis and calcium/calcineurin signaling components have been characterized in C. albicans [15-21]. The calcium/calcineurin signaling functions in ion homeostasis, cell wall biogenesis, morphogenesis and drug resistance in $C$. albicans [22-24]. C. albicans cells lacking calcineurin show significantly reduced virulence in a murine model of systemic infection and fail to survive in the presence of membrane stress [25-27]. However, C. albicans cells lacking $C a C R Z 1$, the major target of calcineurin, are partially virulent in the CAF4-2 strain background and even not virulent in the BWP17 background in the mouse model of systemic infection [28, 29]. Therefore, other targets are responsible for the calcineurinmediated virulence in $C$. albicans. We have recently screened the GRACE (gene replacement and conditional expression) library of 2358 conditional mutants and identified a total of 21 genes whose conditional repression leads to the sensitivity of $C$. albicans cells to high levels of extracellular calcium [30-32]. In addition to 3 reported genes, CRZ1, MIT1 and RCH1 [16, 20, 28, 33], the rest newly-identified 18 calcium tolerance-related genes are involved in tricarboxylic acid cycle, cell wall integrity pathway, cytokinesis, $\mathrm{pH}$ homeostasis, magnesium transport, and DNA damage response.

Microarray analysis indicates that calcium-induced upregulation of 60 genes with a putative CaCrz1-binding motif [5'-G(C/T)GGT-3'] is dependent on both calcineurin and CaCrz1 in C. albicans [28]. Both microarray and RNA sequencing are used to measure genome-wide transcriptomic changes in different organisms, and they complement to each other in transcriptome profiling [34-36]. However, RNA sequencing approach is much more sensitive than the microarray, with the dynamic range of the former reaching at least 8000-fold in comparison to the latter only at around 60-fold in expression levels of genes detected [37].Therefore, we have examined the regulatory function of CaCrz1 in gene expression with the RNA sequencing technology in this study. We show that expression of 219 genes is positively controlled, and expression of 59 genes is negatively controlled, by CaCrz1 in the SN148 background in response to calcium stress. Furthermore, we have revealed an additional CaCrz1-binding motif in promoters of its target genes and demonstrated that CaCrz1 binds to both motifs in the promoter of its target gene CaUTR2.

\section{Methods}

Strains and media

C. albicans strains and plasmids used in this study were described in Table 1. Primers used in this study were listed in Additional file 1: Table S1. Strains were grown 
and maintained at $30^{\circ} \mathrm{C}$ in YPD medium or SD medium $(0.67 \%$ yeast nitrogen base without amino acids, $2 \%$ glucose, and auxotrophic amino acids as needed). Chemicals were obtained from Sigma (USA) and Sangon Biotech (Shanghai, China).

\section{Construction of CRISPR mutant for CaCRZ1}

C. albicans strain SN148 was used as the parent strain to construct the CRISPR inactivation mutant for CaCRZ1 through the CRISPR [Clustered Regularly Interspaced Short Palindromic Repeat)/Cas9] approach (Additional file 1: Figure S1). We designed SgRNA primers CRZ1-sgF and CRZ1-sgR near the start codon of CaCRZ1 using the online software Benchling (https://benchling.com/academic) as well as the repair DNA primers CRZ1-RFand CRZ1-RR containing 40-bp homologous regions flanking the SgRNA sequence (Additional file 1 : Figure. S1). Primers CRZ1sgF and CRZ1-sgR were annealed, cut with BsmBI and cloned into the BsmBI site of pV1093 (Additional file 1: Figure S1A-S1B), which generated the recombinant plasmid pV1093-SgRNA. SgRNA sequence in pV1093-SgRNA was confirmed by DNA sequencing. Primers CRZ1-RF and CRZ1-RR were annealed for PCR amplification of the repair DNA fragment of about $100 \mathrm{bp}$. The repair DNA and the recombinant plasmid pV1093-SgRNA linearized by both $S a c I$ and $K p n I$ were used together to transform cells of C. albicans strain SN148 (Additional file 1: Figure S1C). Potential correct CRISPR mutants for $C a C R Z 1$ were detected with diagnostical PstI-digestion of 1-kb PCR products, containing the SgRNA region, amplified with primers CRZ1-CF and CRZ1-CR from genomic DNA samples of transformants (Additional file 1: Figure S1D$\mathrm{S} 1 \mathrm{E})$. Mutated sites in CaCRZ1 alleles in those potential correct CRISPR mutants were further confirmed by DNA sequencing.

\section{DNA manipulation}

To clone the full-length gene CaCRZ1 into the integration vector CIp10 [40], a DNA fragment containing the 758-bp promoter, the 2196-bp open reading frame (ORF) and the 336-bp terminator region of CaCRZ1 was amplified with primers CRZ1-clonF and CRZ1clonR, and cloned between $K p n I$ and $X h o I$ sites in the CIp10, which yielded CIp10-CaCRZ1. To do complementation experiment, the wild type and the $c r z 1 / c r z 1$ mutant strains were integrated with the StuI-linearized plasmids CIp10 or CIp10-CaCRZ1, respectively, as described [41].

To express the His6-tagged CaCrz1 expression plasmid in bacterial cells, we first optimized the codon usage by mutating all five CTG codons in CaCRZ1 to TCT codon (L22S), AGC codon (L24S), TCC codons (L601S, L649S and L686S) (Additional file 1: Fig. S2). The codonoptimized open reading frame (ORF) of CaCRZ1 was artificially synthesized and cloned into the vector pET28a $(+)$, which yielded pET28a $(+)-C R Z 1$ that expressing the codon-optimized and N-terminally Hisx6 tagged fulllength CaCrz1 (His6-CaCrz1) protein. The pET28a(+)CRZ1 was introduced and expressed in BL21(DE3) bacterial cells as described [42-44].

To construct a lac $\mathrm{Z}$ reporter plasmid, the bacterial lac $Z$ gene was first amplified with a pair of primers lacZ_ORF_F(XhoI) and lacZ_ORF_R(KpnI) from the plasmid pGP8 $[15,28]$, and cloned into the $K p n \mathrm{I}$ and XhoI sites of CIp10 to yield CIp10-lacZ. The terminator of CaACT1 was amplified from the SN148 genomic DNA with two primers ACT1_T_F(KpnI) and ACT1_T $\mathrm{R}(\mathrm{KpnI})$, and cloned into the KpnI site of CIp10-lacZ to yield CIp10-lacZ- $\mathrm{T}_{\mathrm{ACT} 1}$. The CaUTR2 promoter was amplified from the SN148 genomic DNA with a pair of primers UTR2_P_F(XhoI) and UTR2_P_R(XhoI) and

Table 1 Strains and plasmids used in this study

\begin{tabular}{|c|c|c|}
\hline Name & Genotype or Description & Source \\
\hline \multicolumn{3}{|l|}{ Strain } \\
\hline SN148 & Mata/a arg4/arg4 leu2/leu2 his1/his1 ura3::imm434/ura3::imm434 & {$[38]$} \\
\hline HHCA184 & SN148 crz1/crz1 ENO1/eno1:: natMX4 & This study \\
\hline HHCA185 & SN148 crz1/crz1 ENO1/eno1:: natMX4 & This study \\
\hline HHCA187 & SN148 crzl/crzl ENO1/eno1:: natMX4 & This study \\
\hline HHCA1 & SN148 RPS1/rps1::Clp10 & This study \\
\hline $\mathrm{HHCA} 2$ & HHCA184 RPS1/rps1::Clp10 & This study \\
\hline HHCA3 & HHCA184 RPS1/rps1::Clp10-CaCRZ1 & This study \\
\hline \multicolumn{3}{|l|}{ Plasmid } \\
\hline pV1093 & $A m p^{R} \mathrm{Nat}^{R}$ & {$[39]$} \\
\hline pV1093-sgCRZ1 & $A m p^{R} \mathrm{Nat}^{R}$ & This study \\
\hline Clp10 & $A m p^{R} U R A$ & {$[38]$} \\
\hline Clp10-sgCRZ1 & $A m p^{R}$ URA & This study \\
\hline
\end{tabular}


cloned into the XhoI site of CIp10-lacZ-T $\mathrm{T}_{\mathrm{ACT} 1}$ to yield CIp10-UTR2-lacZ.

To mutate the putative CaCrz1-binding motif identified in our study, the underlined sequence in the $5^{\prime}$ TCT(-343) CAACGCCTCA(-333)AAA-3' region of CaUTR2 promoter was mutated to be $5^{\prime}-\mathrm{TCT}(-$ 343)TCTAGA(- 333)AAA-3' (we designated this mutation as UTR2 $(\mathrm{H} \Delta)$ ), which contains a $\mathrm{XbaI}$ site. This was accomplished by a fusion PCR strategy. We first amplified the upstream (A) and downstream (B) fragments of the CaUTR2 promoter with two pairs of primers UTR2_exF/ UTR2_(H $\Delta)_{-} \mathrm{R}$ and UTR2_inR/ UTR2_(H $\Delta)_{-} F$, respectively. These two fragments (A and $\mathrm{B}$ ) were then fused by PCR with the two primers UTR2_P_F(XhoI) and UTR2_P_R(XhoI), and cloned into the XhoI site of CIp10-lacZ-T $\mathrm{ACT1}_{1}$ to yield CIp10UTR2(H $\Delta$ )-lacZ. Similarly, to mutate the putative CaCrz1-binding motif identified in the previous study [28], the underlined sequence in the $\left(5^{\prime}\right.$-TTGT(377) GGGCTT(-371)TGA-3' region of CaUTR2 promoter was mutated to be $\left(5^{\prime}-\mathrm{TTGT}(-377)\right.$ TCTAGAT(-371)TGA-3' (we designated this mutation as UTR2(M $\Delta)$ ), which contains a XbaI site. The upstream (C) and downstream (D) fragments of the CaUTR2 promoter were first PCR amplified with two pairs of primers UTR2_exF/ UTR2_(M $\Delta) \_R$ and UTR2_inR/ UTR2_(M $\Delta)$ _F, respectively. These two fragments (C and $\mathrm{D})$ were then fused by PCR with the two primers UTR2_P_F(XhoI) and UTR2_P_R(XhoI), and cloned into the XhoI site of CIp10-lacZ- $\mathrm{T}_{\mathrm{ACT} 1}$ to yield CIp10UTR2(M $\Delta$ )-lacZ. To create the CIp10-UTR2(HM $\Delta$ )lacZ with mutations for both UTR2 $(\mathrm{H} \Delta)$ and $\mathrm{UTR} 2(\mathrm{M} \Delta)$ in the CaUTR2 promoter, the two DNA fragments (A and $\mathrm{D})$ were fused by PCR with primers UTR2_P_F(XhoI)/ UTR2_P_R(XhoI), and cloned into the XhoI site of CIp10-lacZ- $\mathrm{T}_{\mathrm{ACT1}}$. Inserts in all recombinant plasmids were confirmed by DNA sequencing.

\section{RNA sequencing and data analysis}

To identify genes regulated by CaCrz1, the wild type SN148 and its isogenic CRISPR mutant for CaCRZ1 were grown to log-phase at $30^{\circ} \mathrm{C}$ before they were treated with $0.2 \mathrm{M} \mathrm{CaCl}_{2}$ for $2 \mathrm{~h}$. Total RNA samples were extracted Qiagen RNeasy minikit protocol, and RNA integrity was evaluated using an Agilent 2100 Bioanalyzer (Agilent Technologies, USA) as described [45]. RNA-seq libraries were constructed using Illumina's miSEQ RNA Sample Preparation Kit (Illumina Inc., USA). RNA sequencing, data analysis and sequence assembly were performed by the Quebec Genome Innovation Center at McGill University (Montreal, Canada) [31, 38]. Preparation of the paired-end libraries and sequencing were performed following standard Illumina methods and protocols. The mRNA-seq library was sequenced using an Illumina miSEQ sequencing platform. Clean reads from RNA-Seq data were assembled into full-length transcriptome with the reference genome (http://www.candidagenome.org/). Functional categories of genes were carried out by the Munich Information Center for Protein Sequences (MIPS) analysis.

\section{Galactosidase activity assay}

To measure the UTR2 promoter-driven $\beta$-galactosidase activity in the wild type and the $c r z 1 / c r z 1$ mutant, we integrated the StuI-linearized plasmids containing the lac Z reporters for CaUTR2 promoter into the RPS1 locus of these strains as described $[16,28]$. The $\beta$-galactosidase activity was determined using the substrate ONPG as described [46, 47]. Data are mean \pm SD from six independent experiments. Significant differences were analysed by GraphPad Prism version 4.00. $P$ values of $<0.05$ were considered to be significant.

\section{Results}

\section{Construction of the CRISPR mutant for CaCRZ1}

To further study the regulatory functions of CaCrz1 in gene expression, we constructed three independent CRISPR mutants for CaCRZ1 in the SN148 genetic background (Additional file 1: Figure S1A-S1E). These mutants were sensitive to $0.4 \mathrm{M} \mathrm{CaCl}_{2}$, and their calcium sensitivity was suppressed by the specific inhibitor of calcineurin, cyclosporine A. In addition, they were sensitive to $0.05 \%$ SDS, but not to antifungal drugs including clotrimazole, ketoconazole, fluconazole and terbinafine (Additional file 1: Figure S1F). These results agree with previous reports $[21,28,29]$. We chose one of these CRISPR mutants (HHCA184) for our RNA sequencing, and its calcium-sensitive phenotype could be partially reversed by the introduction of the CaCRZ1 gene back to its genome (Fig. 1). To examine if the two mutated CaCRZ1 alleles in the CRISPR mutant (HHCA184) were still able to express the $\mathrm{CaCrz1}$ proteins in C. albicans cells, we chromosomally integrated the HA tag at the Cterminus of CaCrz1 in both the mutant and the wild type strain SN148. Through western blot analysis, we failed to detect the expression of CaCrz1-HA in the mutant, although we detected two forms of CaCrz1-HA proteins in the wild type, which might correspond to the phosphorylated form and dephosphorylated form of CaCrz1 (Fig. 2). Taken together, our data demonstrate that we have successfully constructed the CRISPR mutant for CaCRZ1.

\section{Transcriptomic profiling of cells lacking CaCRZ1}

Next, we carried out transcript profiling for the wild type and the $c r z 1 / c r z 1$ mutant, growing in log phase in YPD medium at $30^{\circ} \mathrm{C}$ in the absence or presence of $0.2 \mathrm{M}$ $\mathrm{CaCl}_{2}$. Transcripts for two alleles of 6211 genes at various 


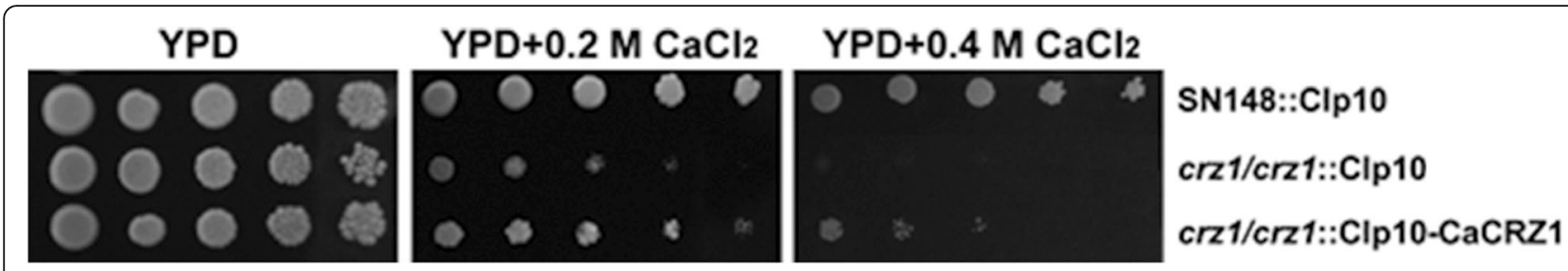

Fig. 1 Phenotypes of CRISPR mutant for CaCRZ1. Cells of the wild-type SN148, the CRISPR mutant and the complemented strain were grown at $30^{\circ} \mathrm{C}$ in liquid YPD overnight, serially diluted by 10 times and spotted on YPD plates with or without supplemented reagents as indicated, respectively. Plates were incubated for $2-5$ days at $30^{\circ} \mathrm{C}$. CsA, cyclosporine $\mathrm{A}$

expression levels were detected in these two strains (SuppInfo 1; GEO Accession number: GSE123122). As compared to the wild type cells without $0.2 \mathrm{M} \mathrm{CaCl}$ treatment, there are 828 genes upregulated in the wild type cells with $0.2 \mathrm{M} \mathrm{CaCl} 2$ treatment, among which 219 genes are positively regulated, and 59 genes are negatively regulated, by CaCrz1 (SuppInfo 2; SuppInfo 3). These genes positively regulated by $\mathrm{CaCrz} 1$ play roles in metabolism (13), cellular transport (23), transcription (7), signal transduction (3), protein fate (17), cell rescue (9), cell cycle (6), cell fate/development/cell type differentiation (14) and cell wall biogenesis (34), with almost half of them (93) being of unknown functions (Table 2). In contrast, these genes negatively regulated by $\mathrm{CaCrz1}$ function in metabolism [20], cellular transport [5], transcription [11] and cell wall biogenesis [3], with one third of them [20] being of unknown functions (Table 3). The CaCRZ1 gene itself is positively regulated by CaCrz1, which is identified in both the previous microarray study and our current study (Table 2).

Among the 219 genes positively regulated by CaCrz1, a total of 40 genes have also been identified by DNA microarray analysis in the previous study (Table 2; 28). Through the online MEME (Multiple Em for Motif Elicitation) software Suite 5.0.2 (http://meme-suite.org/), we scanned promoters of these shared 40 genes and identified a consensus sequence [5'-GGAGGC(G/ A)C(T/A)G-3'], which is different from the putative CaCrz1-binding consensus sequence $\left[5^{\prime}-\mathrm{G}(\mathrm{C} / \mathrm{T}) \mathrm{GGT}\right.$ 3'] previously identified through DNA microarray [28], but similar to $S$. cerevisiae ScCrz1-binding motif [5' GNGGC(G/T)CA-3'] [48]. Therefore, CaCrz1 can bind

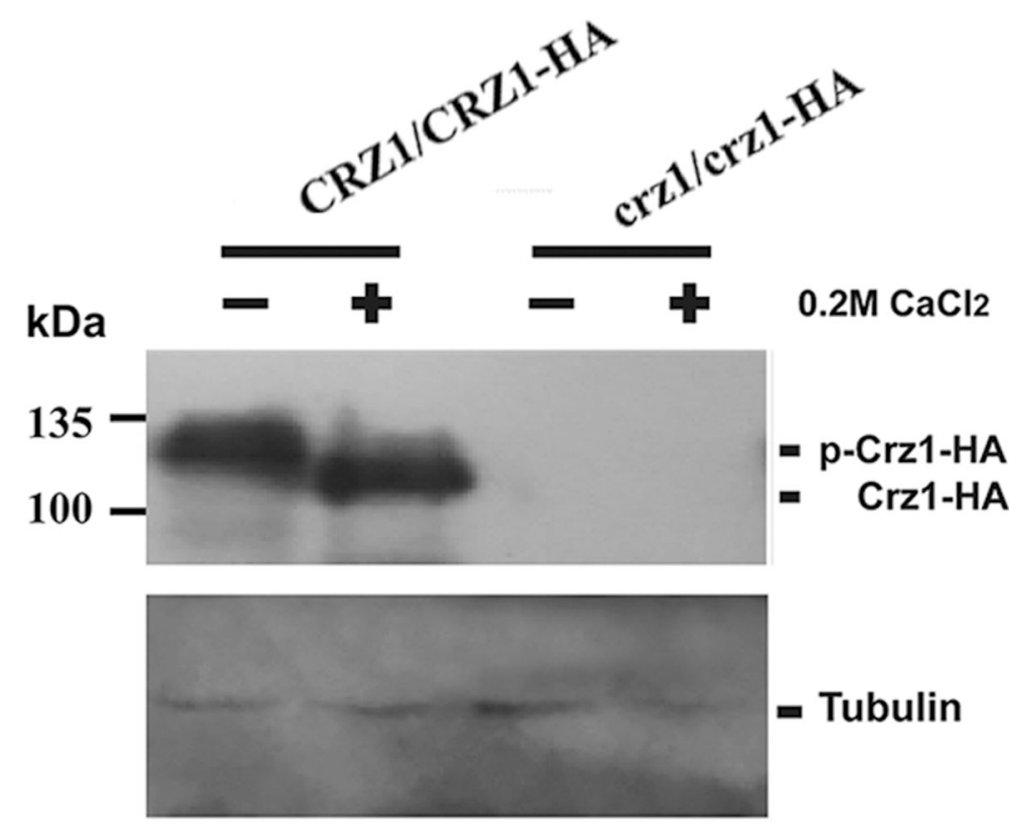

Fig. 2 Expression of the C-terminally HA-tagged CaCrz1 protein in C. albicans cells. The wild type strain SN148 (CRZ1/CRZ1) and the CRISPR mutant for CaCRZ1 (Crzl/Crz1) carrying their chromosomally C-terminally HA-tagged wild-type and mutated CaCRZ1 alleles, respectively, were grown to log-phase in YPD medium at $30^{\circ} \mathrm{C}$ before their cells were collected for total protein extraction. Expression of CaCrz1-HA proteins was detected by Western blot analysis with anti-HA monoclonal antibody. Expression of tubulin was detected using anti-tubulin antibody, which served as an internal expression control 
Table 2 Functional category of 219 genes positively regulated by $\mathrm{CaCrz} 1$ in response to $0.2 \mathrm{M} \mathrm{CaCl} 2$

\begin{tabular}{|c|c|c|c|c|c|c|c|c|c|}
\hline $\begin{array}{l}\text { Systemic } \\
\text { name }\end{array}$ & $\begin{array}{l}\text { Standard } \\
\text { name }\end{array}$ & $\begin{array}{l}\text { Systemic } \\
\text { name }\end{array}$ & $\begin{array}{l}\text { Standard } \\
\text { name }\end{array}$ & $\begin{array}{l}\text { Systemic } \\
\text { name }\end{array}$ & $\begin{array}{l}\text { Standard } \\
\text { name }\end{array}$ & $\begin{array}{l}\text { Systemic } \\
\text { name }\end{array}$ & $\begin{array}{l}\text { Standard } \\
\text { name }\end{array}$ & $\begin{array}{l}\text { Systemic } \\
\text { name }\end{array}$ & $\begin{array}{l}\text { Standard } \\
\text { name }\end{array}$ \\
\hline \multicolumn{10}{|l|}{ Metabolism (13) } \\
\hline C1_04010C & & C2_03640W & UGA11 & C5_00220W & ROT2 & C1_14060W & & C1_11240C & $\mathrm{CHOl}$ \\
\hline C2_01630W & & C2_09150W & MIT1 & CR_00620C & $A R G 1$ & C1_08330C & $\mathrm{ADH} 2$ & C7_02500C & DPP3 \\
\hline C3_05810C & SKN1 & $\underline{\mathrm{C} 1}$ 02590C & $\underline{S N Z 1}$ & C1_01620C & & & & & \\
\hline \multicolumn{10}{|c|}{ Cellular transport (23) } \\
\hline CR_03450W & HXT5 & C5_04440C & SFC1 & C3_03060W & & C3_05270C & HGT5 & C7_02910W & ENA21 \\
\hline C1_09220W & & C2_09770C & INP51 & C1_04630C & & C3_01250W & PMC1 & C7_00100W & FRP2 \\
\hline CR_05310W & & C4_03110W & & C3_07230W & & C1_01100W & $\mathrm{CCH1}$ & C1_09400C & FTH1 \\
\hline C2_03800C & & CR_09170C & SSU1 & C2_06470W & $\underline{\text { RTA2 }}$ & C2_07730W & YVC1 & C1_06610C & HAK1 \\
\hline$\underline{\mathrm{C} 1}$ 06480C & & CR_07100W & $F L C 2$ & CR_09680C & $\underline{\text { RTA4 }}$ & & & & \\
\hline \multicolumn{10}{|l|}{ Transcription (7) } \\
\hline CR_03890W & WOR3 & C7_00970C & YOX1 & C3_05780C & $\underline{C R Z 1}$ & C1_05340C & ZCF2 & C7_04230W & $N R G 1$ \\
\hline CR_02300C & & C4_04210C & $\mathrm{SOH} 1$ & & & & & & \\
\hline
\end{tabular}

Signal transduction (3)
C5_02290W PDE1
C4_06480C CEK1
C7_00360W DFI1

Protein fate (folding, modification, destination) (17)

\begin{tabular}{|c|c|c|c|c|c|c|c|c|c|}
\hline C1_13220C & $\underline{A K R 1}$ & C5_01440C & & C2_00930C & VPS24 & C5_01210W & VPS1 & C4_03890W & PTP2 \\
\hline CR_00290W & & C5_05060C & & C4_04660C & & C3_03870C & SAPG & C4_05810W & \\
\hline C6_03500C & SAP4 & C2_01670C & $\underline{S T T 3}$ & C4_00070C & & C7_03250C & $\underline{P D / 1}$ & CR_00260W & KIN2 \\
\hline C1_08170C & BUL1 & C2_08790W & JEM1 & & & & & & \\
\hline \multicolumn{10}{|l|}{ Cell rescue (9) } \\
\hline CR_06040W & & CR_01730W & IFU5 & C2_02060C & FMO1 & C2_00680C & SOD5 & C1_02700C & \\
\hline C2_09220W & $\underline{D D R 48}$ & C3_00480C & DOT5 & C2_05660W & PNG2 & CR_05390W & PST3 & & \\
\hline \multicolumn{10}{|l|}{ Cell Cycle (6) } \\
\hline C1_09870W & HCM1 & C6_03260W & & C1_05170C & CUE5 & C1_08570C & PCL2 & C3_03850C & SOL1 \\
\hline C5_01680C & CCN1 & & & & & & & & \\
\hline \multicolumn{10}{|c|}{ Cell fate/development/cell type differentiation (14) } \\
\hline C4_03510C & HWP2 & C7_00120W & & C1_00850W & IHD2 & C2_03040W & $P L C 2$ & C3_05190C & MCA1 \\
\hline C2_07930C & VRP1 & C1_07770W & FGR6-3 & C4_00600C & MUCl & C2_05260W & BUD14 & C3 $05710 \mathrm{~W}$ & $\underline{R C T 1}$ \\
\hline C6_00940C & & C2_00080C & FAV3 & $\underline{\text { C4_01010C }}$ & $\underline{D A G 7}$ & C1_01440C & POX18 & & \\
\hline \multicolumn{10}{|c|}{ Cell wall biosynthesis (34) } \\
\hline C5_02630C & MNN1 & $\underline{\text { CR_00740C }}$ & $\underline{B M T 3}$ & $\underline{\text { C3_01730C }}$ & $\underline{\text { UTR2 }}$ & $\underline{C R \_04440 C}$ & $\underline{R B R 1}$ & C3_02140C & \\
\hline C4_06540W & MNN4 & C2_01560W & BMT5 & CR_10480W & PGA1 & $\underline{\text { C5_02460C }}$ & $\underline{E C M 331}$ & C2_00520W & DFG5 \\
\hline C1_04900W & $\underline{\text { MNN15 }}$ & C3_03450C & BMT7 & C1_09080C & $\underline{P G A 6}$ & CR_03790C & KRE 1 & $\underline{\text { C4_02720C }}$ & \\
\hline C2_01300C & MNN24 & CR_00180C & CHT1 & CR_08510W & PGA13 & C6_01690W & ACF2 & $\underline{\text { C6 }}$ 02730W & $\underline{S A C 6}$ \\
\hline C2_03690C & MNN42 & C2_02010C & CHT4 & CR_02280W & $\underline{P G A 23}$ & C5_04110W & SCW11 & C2_05040C & \\
\hline C4_06990W & MNN46 & CR_09020C & CHS2 & CR_04900C & PGA39 & C1_00220W & PHR2 & C4_05100C & MYO5 \\
\hline C3_01830C & MNT2 & C4_02900C & $\mathrm{CRH} 11$ & C2_00100C & PGA52 & C1_04000C & KTR4 & & \\
\hline \multicolumn{10}{|l|}{ Jnknown (93) } \\
\hline CR_00380W & & C3_07360W & DLD2 & CR_10570C & YHB4 & C3_04100W & & C4_03590C & $\mathrm{OSH} 3$ \\
\hline C4_00410W & & C3_07470W & & C1_11970C & & C2_09050C & & C5_04330W & \\
\hline C2_08620W & & C4_06470W & & C1_12060C & & C2_10150W & & C5_04470C & \\
\hline
\end{tabular}


Table 2 Functional category of 219 genes positively regulated by $\mathrm{CaCrz1}$ in response to $0.2 \mathrm{M} \mathrm{CaCl2}$ (Continued)

\begin{tabular}{|c|c|c|c|c|c|c|c|c|c|}
\hline $\begin{array}{l}\text { Systemic } \\
\text { name }\end{array}$ & $\begin{array}{l}\text { Standard } \\
\text { name }\end{array}$ & $\begin{array}{l}\text { Systemic } \\
\text { name }\end{array}$ & $\begin{array}{l}\text { Standard } \\
\text { name }\end{array}$ & $\begin{array}{l}\text { Systemic } \\
\text { name }\end{array}$ & $\begin{array}{l}\text { Standard } \\
\text { name }\end{array}$ & $\begin{array}{l}\text { Systemic } \\
\text { name }\end{array}$ & $\begin{array}{l}\text { Standard } \\
\text { name }\end{array}$ & $\begin{array}{l}\text { Systemic } \\
\text { name }\end{array}$ & $\begin{array}{l}\text { Standard } \\
\text { name }\end{array}$ \\
\hline C1_03870C & & C5_03970W & & C1_13240W & & C2_10160W & & C5_04480C & \\
\hline CR_07160C & & C2_08960C & & C1_13590W & & C2_10720C & & C5_04540C & \\
\hline C1_08610C & & C1_00760W & & C1_13810W & & C3_02710W & & C6_01250W & \\
\hline C3_01550C & TOS1 & C1_01510W & & C2_00110W & & C3_04190W & & C6_02210W & \\
\hline C5_04960W & & C1_02370C & & C2_00130W & & C3_06670C & & C6_04420W & \\
\hline C7_01700W & & C1_04440W & & C2_00750W & & C3_06680C & & C7_00310C & \\
\hline C1_03150C & & C1_04470C & & C2_00920W & & C4_04190C & & C7_00350C & \\
\hline C1_09800C & TVP18 & C1_05450W & & C2_00940W & & C4_04200C & & C7_01390W & \\
\hline C2_03020C & & C1_05920W & & C2_02220C & & C4_05000W & & C7_02370W & \\
\hline CR_00420W & & C1_07990C & & C2_02900W & & C4_05250W & & C7_03310W & \\
\hline CR_07480W & & C1_08830C & & C2_02910W & & C4_05800C & & CR_01020C & \\
\hline CR_08470W & & C1_10060C & & C2_03570C & & C4_07260W & & CR_02880W & \\
\hline C4_00860C & & C1_10580C & & C2_04750W & & C5_00410W & & CR_03780C & \\
\hline C3_02570W & & C1_11260C & & C2_05120C & & C5_03430W & & CR_06550C & \\
\hline C4_03870C & & C1_11270W & & C2_06630C & & C5_04030W & & CR_08990C & \\
\hline CR_05460W & & C4_00980C & MRV1 & C2_08910C & & & & & \\
\hline
\end{tabular}

\#Underlined genes are shared CaCrz1-regulated genes that has been identified by DNA microarray in a previous study [28]. Shadowed genes are sequence homologues for $9 \mathrm{~S}$. cerevisiae genes positively regulated by $\mathrm{ScCrz} 1$ in response to $0.2 \mathrm{M} \mathrm{CaCl} 2$ reported in a previous study [48].-

to two different CaCrz1-binding motifs in promoters of its target genes. This has also been reported previously for M. oryzae MoCrz1 [49, 50].

\section{CaCrz1 binds in vitro and in vivo to two putative binding motifs in the promoter of CaUTR2}

Base on the consensus motif [5'-GGAGGC(G/A)C(T/A)G3'] from the MEME analysis described above, we found one putative CaCrz1 binding motif, the 5'-TGAGGC GTTG-3' region in the complementary sequence of the 5 'C(-342)AACGCCTCA(-333)-3' site in the promoter of one of the CaCrz1 target genes, CaUTR2 (Fig. 3a). Next, we tested the roles of this motif and the other putative CaCrz1 binding motif, 5'-G(-376)GGCT(-372)-3', which was identified previously [28].

Different from other eukaryotes, C. albicans does not follow the universal genetic code, by translating the CTG codon into serine instead of leucine [51]. Therefore, we first optimized the codon usage by mutating all five CTG codons in CaCRZ1 to TCT codon (L22S), AGC codon (L24S), TCC codons (L601S, L649S and L686S) (Additional file 1: Figure S2). The codonoptimized and Hisx6 tagged full-length CaCrz1 (His6CaCrz1) was expressed in bacterial cells and purified (Additional file 1: Figure S3). Electrophoretic mobility shift (EMSA) assay showed that His6-CaCrz1 bound to both the P1 probe containing the putative binding motif identified in our study (Lane 2), the P2 probe containing the putative binding motif identified in the previous study [28] (Lane 5), and the Probe 3 containing two of the motifs (Lanes 8) (Fig. 3b). The binding of His6CaCrz1 to Probe 1, Probe 2 and Probe 3 was abolished by their specific competitors, unlabelled probes, respectively (Lanes 3, 6 and 9) (Fig. 3b). Taken together, these results demonstrate that $\mathrm{CaCrz1}$ can indeed bind in vitro to both motifs in the CaUTR2 promoter.

To examine if CaCrz1 binds to the CaUTR2 promoter region in vivo, we carried out chromatin immunoprecipitation (ChIP) experiments. We examined the wild-type SN148 strain expressing a chromosomally and C-terminally HA-tagged CaCrz1 (CaCrz1-HA) under the control of the CaCRZ1 promoter (left two lanes in Fig. 3c), and the wild-type SN148 strain with the untagged wild type CaCrz1 and with the CIp10 vector integrated as the control (right two lanes in Fig. 3c). DNA samples isolated from their anti-HA chromatin immunoprecipitates were used in PCR assays to detect CaCrz1-HA target promoters (The second and the third lanes in Fig. 3c). As controls, their whole-cell extracts (WCEs) were used in parallel PCR assays to ensure the equivalence of the IP starting materials (The first and the fourth lanes in Fig. 3c). We found that the promoter region containing two putative binding motifs in the CaUTR2 promoter were enriched in the anti-HA IPs of the CaCrz1-HA strain (The second lane in Fig. 3c), but not in the untagged 
Table 3 Functional category of 59 genes negatively regulated by $\mathrm{CaCrz} 1$ in response to $0.2 \mathrm{M} \mathrm{CaCl} 2$

\begin{tabular}{|c|c|c|c|c|c|c|c|c|c|}
\hline Systemic name & Standard name & Systemic name & Standard name & Systemic name & Standard name & Systemic name & Standard name & Systemic name & Standard name \\
\hline \multicolumn{10}{|l|}{ Metabolism (20) } \\
\hline C6_00760W & & C1_13870W & MET3 & C4_00490W & & C1_03820W & PDR16 & CR_05340C & IFE2 \\
\hline C6_00620W & FCA1 & C7_00490C & & C5_05150C & & C4_06950W & & & \\
\hline C7_01600W & & C1_04880C & MRPL37 & C7_00950W & YML6 & C3_02030W & & C4_04820C & \\
\hline C7_01440W & & CR_01390W & MGE1 & C3_05440C & & C7_02120C & & C7_01020C & \\
\hline \multicolumn{10}{|l|}{ CR_10120C } \\
\hline \multicolumn{10}{|c|}{ Cellular transport (5) } \\
\hline CR_02920C & AQY1 & C6_03790C & HGT10 & C2_01020W & HGT6 & C6_04610C & NAG3 & C6_03390W & \\
\hline \multicolumn{10}{|l|}{ Transcription (11) } \\
\hline C4_05880W & GAT1 & C2_00280C & & CR_10690W & POP3 & CR_02030C & & CR_01710W & \\
\hline C2_09460C & & C2_05230C & RPF2 & C5_01480W & FYV5 & C6_02910W & POP4 & C6_01040C & \\
\hline C5_00980W & TRY3 & & & & & & & & \\
\hline \multicolumn{10}{|c|}{ Cell wall biogenesis (3) } \\
\hline CR_04420C & $R B R 2$ & CR_01930C & BlO2 & C4_00720W & $\operatorname{CSP} 2$ & & & & \\
\hline \multicolumn{10}{|l|}{ Unknown (20) } \\
\hline CR_09350C & & C2_06440C & & C6_0072OC & $\operatorname{cox} 15$ & C5_01785W & & CR_06330C & \\
\hline C1_11320C & & C3_00120W & & CR_06920W & & C4_03300C & & C1_00970W & \\
\hline C3_03490W & RSN1 & C3_04510W & & C1_04600C & & C1_14480W & & C3_00410C & \\
\hline C4_06960W & & C1_10500W & & C1_09820C & & C7_03210W & & C1_10250C & \\
\hline
\end{tabular}

CaCrz1 strain (The third lane in Fig. 3C). Together, these data demonstrate that CaCrz1 binds in vivo to the promoter region containing the two motifs of CaUTR2.

Mutations of two putative binding motifs in the promoter abolish the CaCrz1-regulated expression of CaUTR2

To further characterize the effects of two CaCrz1-binding motifs on the expression of CaUTR2, we generated four plasmids, CIp10-UTR2-lacZ, CIp10-UTR2(H $\Delta$ )-lacZ, CIp10-UTR2(M $\Delta$ )-lacZ and CIp10-UTR2(HM $\Delta$ )-lacZ, containing the wild-type CaUTR2 promoter, the singlemotif mutated promoter UTR2(H $\Delta)$, the single-motif mutated promoter UTR2 $(\mathrm{M} \Delta)$ and the double-motif mutated promoter UTR2(HM $\Delta)$. In the absence of supplemented calcium, a basal expression level was detected for the wild type promoter UTR2-lacZ in the wild type cells (Fig. 4a). As expected, in response to $0.2 \mathrm{M} \mathrm{CaCl}_{2}$, the $\beta$ galactosidase activity of the wild type promoter UTR2-lacZ was increased by more than two times in the wild-type cells, but did not change significantly in the $\mathrm{crz} 1 / \mathrm{crz} 1 \mathrm{mu}-$ tant cells (Fig. 4a). This indicates that the calcium-induced expression of CaUTR2 is dependent on CaCrz1.

As compared to the wild-type promoter UTR2(H $\Delta)$, the $\beta$-galactosidase activities of two single mutated promoters UTR2 $(\mathrm{H} \Delta)$ and UTR2 $(\mathrm{M} \Delta)$ were significantly reduced in the absence or presence of $0.2 \mathrm{M} \mathrm{CaCl}_{2}$ in the wild type cells (Fig. 4b). The $\beta$-galactosidase activity of the double mutated promoter UTR2(HM $\Delta$ ) were even further reduced than those of two single mutated promoters UTR2 $(\mathrm{H} \Delta)$ and $\operatorname{UTR} 2(\mathrm{M} \Delta)$ in the absence or presence of $0.2 \mathrm{M} \mathrm{CaCl}_{2}$ in the wild type cells (Fig. $4 \mathrm{~b}$ ). Taken together, these results suggest that two CaCrz1binding motifs play additive roles in the regulation of CaUTR2 expression.

\section{CaCrz1 binds in vitro and in vivo to its own promoter}

Both a previous study and our current study have observed that CaCRZ1 itself is positively regulated by CaCrz1 (Table 2; 28). Base on the consensus motif [5'GGAGGC(G/A)C(T/A)G-3'] identified in our study, we identified two putative $\mathrm{CaCrz} 1$ binding motif, the $5^{\prime}$-T(-519)GAGGGACTG(-528)-3' site (within the Probe 1 sequence) and the $5^{\prime}$-G(-446)GGGGGTCTG(455)-3' site (within the Probe 2 sequence) in the complementary sequence, in its own promoter (Fig. 5a). Based on the consensus motif [ $\left.5^{\prime}-\mathrm{G}(\mathrm{C} / \mathrm{T}) \mathrm{GGT}-3^{\prime}\right]$ identified previously [28], we also identified one putative CaCrz1 binding motif, the $5^{\prime}-\mathrm{G}(-368) \mathrm{TGGT}(-372)-3^{\prime}$ site (within the Probe 3 sequence), in the complementary sequence of CaCRZ1 promoter (Fig. 5a). The fourth putative CaCrz1 binding motif, the $5^{\prime}-\mathrm{C}(-84) \mathrm{TGGT}(-80)-3^{\prime}$ site (within the Probe 4 sequence) was identified previously [28].

EMSA assay demonstrated that His6-CaCrz1 bound to only the P1 probe (Lane 2), but not to other three probes, Probe 2 (Lane 5), Probe 3 (Lane 8) and Probe 4 (Lane 11) (Fig. 5b). The binding of His6-CaCrz1 to Probe 1 was abolished by its specific competitor, unlabelled Probe 1 (Lane 3) (Fig. 5b). ChIP analysis indicated that the promoter region containing the $5^{\prime}-\mathrm{T}(-$ 


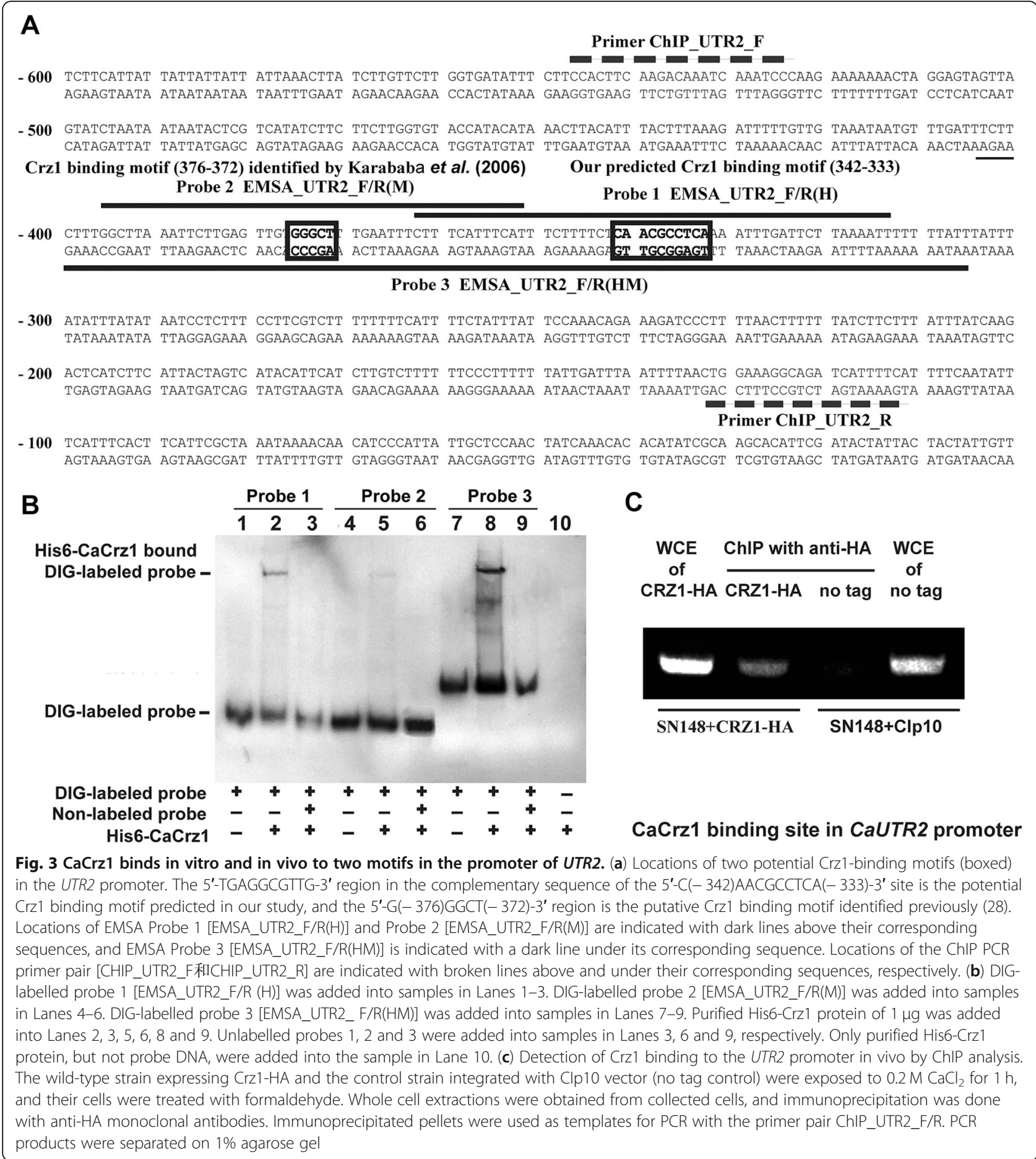

519)GAGGGACTG(-528)-3' site (within the Probe 1 sequence) was enriched in the anti-HA IPs of the CaCrz1-HA strain (Lane 2), but not in the untagged CaCrz1 strain (lane 3) (Fig. 5c). These results demonstrate that $\mathrm{CaCrz1}$ regulates its own expression by binding to the motif $5^{\prime}-\mathrm{T}(-519)$ GAGGGACTG(-528)-3' in its own promoter. The autoregulation phenomenon of this transcription factor gene has also been previously shown in the rice blast pathogen $M$. oryzae MoCrz1 $[49,50]$.

\section{Discussion}

Microarrays are based on the hybridization of oligonucleotide DNA sequences, representing the entire set of genes of an organism arranged in a grid pattern, with 

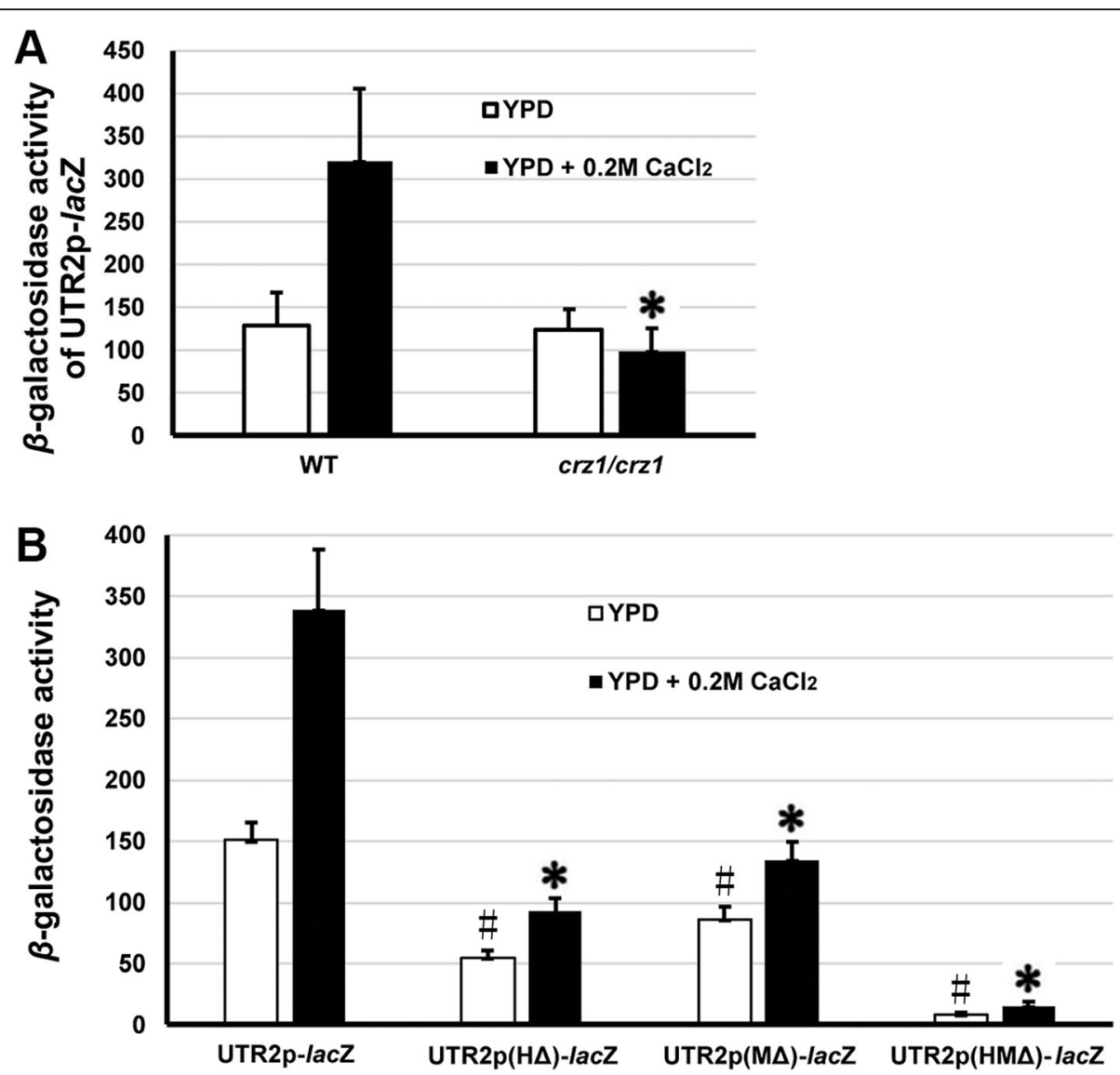

Fig. 4 Two CaCrz1-binding motifs in the promoter play additive roles in the regulation of CaUTR2 expression. (a), $\beta$-galactosidase activities of the wild-type promoter UTR2-lacZ in the wild-type SN148 and the $\mathrm{crz} 1 / \mathrm{crz} 1$ mutant cells in the absence or presence of $0.2 \mathrm{M} \mathrm{CaCl} 2$. The asterisk (*) indicates statistically significant differences $(P<0.05)$ in the $\beta$-galactosidase activity between the wild type strain SN148 and the $\mathrm{crzl} / \mathrm{crz} 1$ mutant strain in the absence or presence of $0.2 \mathrm{M} \mathrm{CaCl}_{2}$, respectively. (b), $\beta$-galactosidase activities of the wild-type promoter UTR2-lacZ, two single mutated promoters UTR2(H $\Delta$ )-lacZ and UTR2(M $\Delta$ )-lacZ as well as the double mutated promoter UTR2(HM $\Delta$ )-lacZ in the wild-type SN148 cells in the absence or presence of $0.2 \mathrm{M} \mathrm{CaCl}_{2}$. The asterisks $\left(^{*}\right)$ and $\left(^{*}\right)$ indicate statistically significant differences $(P<0.05)$ in the $\beta$ galactosidase activity between the wild type promoter and each of the mutated promoters in the wild-type strain SN148 in the absence or presence of $0.2 \mathrm{M} \mathrm{CaCl}_{2}$, respectively

complementary DNA (cDNA) molecules derived from the transcriptome in a cell sample, while cDNA molecules derived from a sample are directly and massively sequenced in the case of RNA-sequencing methodology $[52,53]$. As compared to microarrays, RNA sequencing technology offers increased specificity and sensitivity, but the application of multiple transcriptome measurement methods can improve the comprehension of the global gene expression profile of one organism [34, 35]. Through RNA sequencing, we have identified 219 genes positively, and 59 genes negatively, regulated by CaCrz1 in response to calcium stress in C. albicans. A total of 40 out of the 219 genes identified in this study to be positively regulated by CaCrz1 account for the majority of 60 genes identified by DNA microarray analysis in the previous study (Table 2; 28). Therefore, our current study has expanded the global expression profile of genes controlled by $\mathrm{CaCrz} 1$ in response to calcium stress in C. albicans. This provides a basis for further understanding the regulation of calcium homeostasis in this important human fungal pathogen.

In addition to the CaCrz1-binding motif (M) identified in the previous study [28], we have revealed a novel CaCrz1-binding motif $(\mathrm{H})$ through the MEME analysis of promoters of 40 common genes identified to be controlled by $\mathrm{CzCrz1}$ through both RNA sequencing and microarray approaches (Fig. 3). Furthermore, we have demonstrated that $\mathrm{CaCrz1}$ binds in vitro and in vivo to these two motifs in the promoter of its target gene CaUTR2, and that these two calcineurin-dependent response elements (CDREs) might play additive roles in the regulation of CaUTR2 expression (Fig. 6). Similarly, two MoCrz1-binding motifs in promoters of target genes have been demonstrated in the rice fungal pathogen $M$. oryzae [49]. Among the 219 genes positively regulated by $\mathrm{CaCrz1}$, we found that promoters of 79 genes contain 


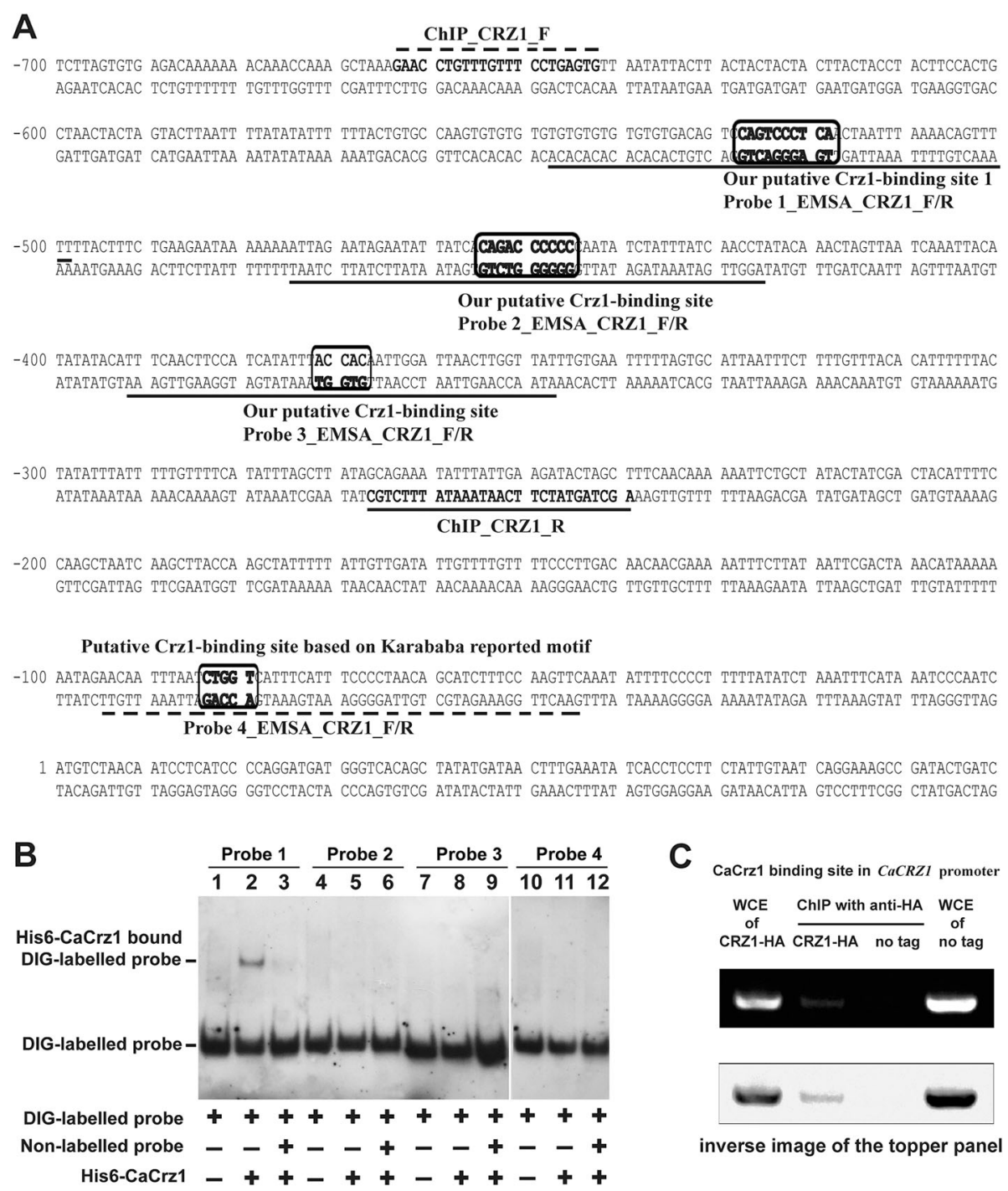

Fig. 5 CaCrz1 binds in vitro and in vivo to its own promoter. (a) Locations of three predicated CaCrz1-binding motifs (boxed and within Probe 1, Probe 2 and Probe 3 sequences) based on the consensus motif we discovered in this study and one predicated CaCrz1-binding motif (boxed and within the Probe 4 sequence). Locations of the ChIP PCR primer pair [CHIP_CRZ1_F和CHIP_CRZ1_R] are indicated with broken lines above and under their corresponding sequences, respectively. (b) DIG-labelled Probe 1_EMSA_CRZ1_F/R was added into samples in Lanes 1-3. DIG-labelled Probe 2_EMSA_CRZ1_F/R was added into samples in Lanes 4-6. DIG-labelled Probe 3_EMSA_CRZ1_F/R was added into samples in Lanes 7-9, and DIG-labelled Probe 4_EMSA_CRZ1_F/R was added into samples in Lanes 10-12. Unlabelled probes 1, 2, 3 and 4 were added into samples in Lanes 3, 6, 9 and 12, respectively. Purified His6-Crz1 protein of $1 \mu \mathrm{g}$ was added into Lanes 2, 3, 5, 6, 8, 9, 11 and 12. (C) Detection of CaCrz1 binding to its own promoter in vivo by ChIP analysis. The same pair of strains were treated and their whole cell extracts were immunoprecipitated as in Fig. 3C. PCR reactions were carried out with ChIP primers CHIP_CRZ1_F和CHIP_CRZ1_R. The lower panel is the inverse image of the topper panel, which is for a better view of the PCR band in the second lane

both motifs $(\mathrm{M}$ and $\mathrm{H})$, promoters of 59 genes contain only motif $\mathrm{H}$, promoters of 45 genes contain only motif $\mathrm{M}$, and promoters of 36 genes contain neither motif $\mathrm{H}$ or motif M (Additional file 2). Therefore, expression of target genes seems to be differentially regulated by CaCrz1.

In S. cerevisiae, 125 calcium-specific and calcineurindependent genes reported in a previous study [48]. Out of these 125 genes, there are 83 genes that are positively regulated by ScCrz1 (Additional file 3). From the $C$. albicans database (http://www.candidagenome. org/), we were able to find 38 C. albicans homologs for these ScCrz1-dependent S. cerevisiae genes, but only 9 of these 38 C. albicans homologs are present in the list of genes identified in this study to be CaCrz1dependent (Table 2; Additional file 3). Therefore, target genes of ScCrz1 and CaCrz1 seem to be very divergent. This is supported by our observation that the amino 


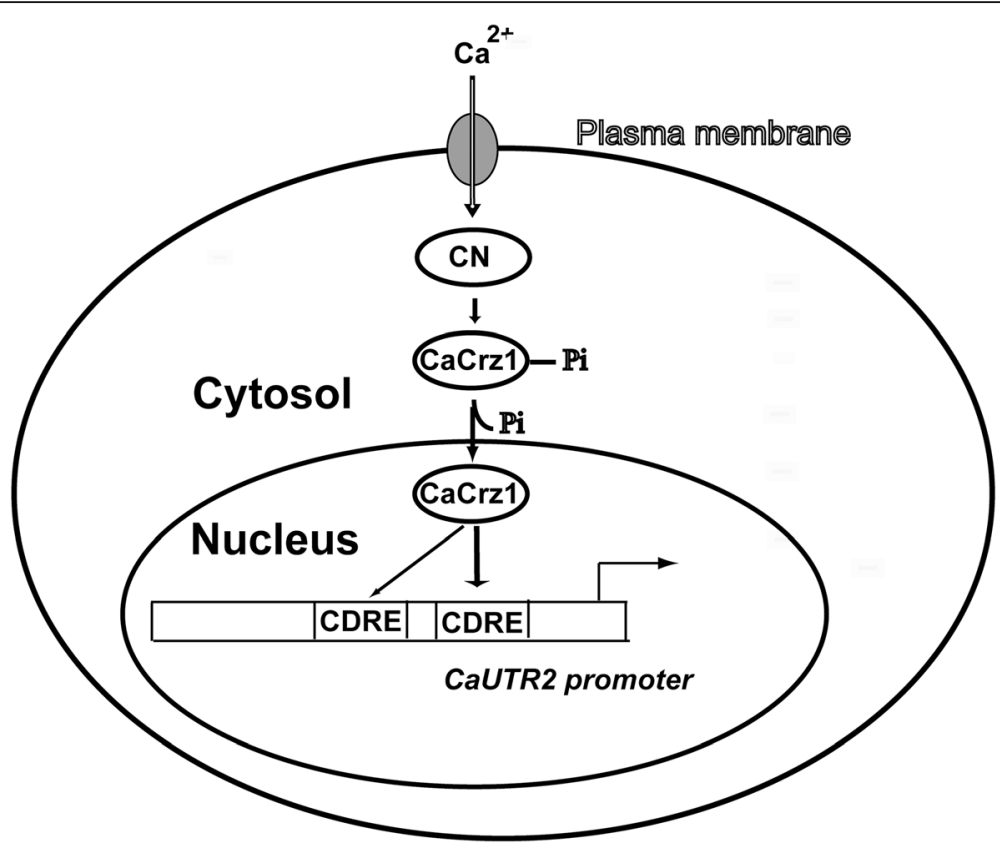

Fig. 6 Schematic model for the regulation of CaUTR2 expression by the transcription factor CaCrz1 in response of C. albicans cells to extracellular calcium stress. Influx of extracellular calcium ions to the cytosol leads to the activation of calcineurin, which in turn dephosphorylates and activates CaCrz1. Dephosphorylated CaCrz1 enters to the nucleus to bind to two CaCrz1 binding motifs (calcineurin dependent response element; CDRE) in the promoter of CaUTR2, which results in the activation of CaUTR2 expression

acid sequences of ScCrz1 and CaCrz1 shares only 31.9 and $24 \%$ similarity and identity, respectively, although their predicted structures are very similar (Fig. S4 in Additional file 1). Similar to the homologs in S. cerevisiae, M. oryzae and another human fungal pathogen Aspergillus fumigatus [49], expression of PMC1 (C3_ 01250W_A) and RCT1 (C3_05710W) is positively controlled by CaCrz1, although expression of RCN1 (C6 01160W_A) is not regulated by CaCrz1 (SuppInfo 1 and 2; GEO Accession number: GSE123122). This is consistent with previous observations on Cryptococcus neoformans CBP1, the homolog of $R C N 1$, that neither is regulated by nor interacts with Crz1 in this human fungal pathogen [54, 55]. In contrast, expression of $R C N 1$ is regulated by Crz1 in S. cerevisiae, M. oryzae and another human fungal pathogen Aspergillus fumigatus, which forms a feedback mechanism for the regulatory role of Rcn1 as an inhibitor of calcineurin [48, $55,56]$. Nevertheless, overexpression of C. albicans RCN1 could inhibit S. cerevisiae calcineurin function [21]. Taken together, these data indicate that regulation of the calcium/calcineurin signaling pathway is diverged in fungal pathogens, although the core calcium signaling machinery (calmodulin, calcineurin and Crz1) is highly conserved across these species. This is consistent with the previous hypothesis $[49,56,57]$.

It is interesting to note that the calcium-sensitive phenotype of the CRISPR mutant for CaCRZ1 could only be partially reversed by the introduction of the fulllength CaCRZ1 gene back to its genome (Fig. 1). Transcripts of the CRISPR mutant CaCRZ1 from the CaCRZ1 locus might compete with those of the wildtype CaCRZ1 transcripts derived from CIp10-CaCRZ1 at the CaRPS1 locus, which might interfere with the translational efficiency of wild-type $C a C R Z 1$ transcripts. This might explain the partial complementation of calcium sensitivity of the CRISPR mutant for CaCRZ1 by CIp10-CaCRZ1. Furthermore, the full-length $6 x \mathrm{His}$ tagged $\mathrm{CaCrz} 1$ protein expresses in bacterial cells as a protein of about $100 \mathrm{kDa}$ (Additional file 1: Figure S3), which is much bigger than its predicted size $(=80 \mathrm{kDa})$. However, the dephosphorylated form of CaCrz1 expressed in C. albicans cells in response to calcium stress also shows a molecular weight of more than 100 $\mathrm{kDa}$ (Fig. 2), which is similar to that of CaCrz1 expressed in bacterial cells. Therefore, this mobility shift could be due to the conformation of CaCrz1 itself, but not the host cell environment or the tag type or tag location (N-terminus or C-terminus).

\section{Conclusions}

In this study, through RNA sequencing we have identified 219 genes that are positively, and 59 genes that are negatively, controlled by CaCrz1 in response to calcium stress. We have also revealed and demonstrated experimentally a novel consensus CaCrz1-binding motif [5'- 
GGAGGC(G/A)C(T/A)G-3'] in promoters of CaCrz1 target genes. In addition, CaCrzl binds to its own promoter and shows an autoregulatory mechanism for CaCRZ1 expression. These findings would contribute to our further understanding of molecular mechanisms regulating calcium homeostasis.

\section{Supplementary information}

Supplementary information accompanies this paper at https://doi.org/10. 1186/s12964-019-0473-9.

\section{Video abstract}

Additional file 2: Figure S1. Construction and phenotypes of CRISPR mutants for CaCRZ1. Figure S2. Alignment between the amino acid sequences of the wild type and the codon optimized version of CaCrz1. Figure S3. Expression and purification of the codon optimized and His6tagged $\mathrm{CaCrz1}$ protein in bacterial cells. Figure S4. Differences between CaCrz1 and ScCrz1. Table S1. Primers used in this study.

Additional file 3. Promoter analysis of 219 genes whose expression is positively regulated by $\mathrm{CaCrz1 \#}$

Additional file 2: Figure S1. Construction and phenotypes of CRISPR mutants for CaCRZ1. Figure S2. Alignment between the amino acid sequences of the wild type and the codon optimized version of CaCrz1. Figure S3. Expression and purification of the codon optimized and His6tagged $\mathrm{CaCrz1}$ protein in bacterial cells. Figure S4. Differences between CaCrzl and ScCrz1. Table S1. Primers used in this study.

Additional file 3. Promoter analysis of 219 genes whose expression is positively regulated by $\mathrm{CaCrz} 1 \#$

Additional file 4. Comparison of calcium-specific and Crz1-dependent genes in Saccharomyces cerevisiae and Candida albicans.

\section{Abbreviations}

ChIP: Chromatin immunoprecipitation; CRISPR: Clustered regularly interspaced short palindromic repeat; $\mathrm{Crz1}$ : Calcineurin-responsive zinc finger 1; EMSA: Gel electrophoretic mobility shift assay; MEME: Multiple em for motif elicitation; NFAT: the nuclear factor of activated T cells; PCR: Polymerase chain reaction; YPD: Yeast peptone dextron

\section{Acknowledgements}

We gratefully acknowledge the help of Malcolm Whiteway' laboratory members for technical support.

\section{Ethical approval and consent to participate}

N/A

\section{Authors' contributions}

HX, TF and ORP performed the experiments. $L J$ designed the study and wrote the manuscript. $\amalg$ and MW analyzed the data. All authors read and approved the final manuscript.

\section{Authors' information}

N/A

\section{Funding}

This work was funded by the National Natural Science Foundation of China to $\amalg$ (No. 81571966 and No. 81371784) and the NSERC Discovery to MW (RGPIN/4799) and the CRC to MW (950-228957).

\section{Availability of data and materials}

All data generated or analyzed during this study are included in this published article and deposited in the database Gene Expression Omnibus (GEO) site.

\section{Consent for publication}

All authors approved the final manuscript.

\section{Competing interests}

The authors declare that they have no competing interests.

\section{Author details}

${ }^{1}$ Laboratory for Yeast Molecular and Cell Biology, Department of Food Science, School of Agricultural Engineering and Food Science, Shandong University of Technology, Zibo 255000, China. ${ }^{2}$ Department of Biology, Concordia University, Montreal, Quebec H4B 1R6, Canada.

Received: 12 September 2019 Accepted: 25 October 2019

Published online: 03 January 2020

\section{References}

1. Tang RJ, Luan S. Regulation of calcium and magnesium homeostasis in plants: from transporters to signaling network. Curr Opin Plant Biol. 2017:39:97-105.

2. Bond $\mathrm{R}$, Ly $\mathrm{N}$, Cyert MS. The unique $\mathrm{C}$ terminus of the calcineurin isoform CNAß1 confers non-canonical regulation of enzyme activity by $\mathrm{Ca}^{2+}$ and calmodulin. J Biol Chem. 2017;292:16709-21.

3. Espeso EA. The CRaZy calcium cycle. Adv Exp Med Biol. 2016;892:169-86.

4. Plattner $H$, Verkhratsky $A$. The ancient roots of calcium signalling evolutionary tree. Cell Calcium. 2015;57:123-32.

5. Medler KF. Calcium signaling in taste cells: regulation required. Chem Senses. 2010;35:753-65.

6. Serra-Cardona A, Canadell D, Arino J. Coordinate responses to alkaline $\mathrm{pH}$ stress in budding yeast. Microb Cell. 2015;2:182-96.

7. Cui J, Kaandorp JA, Sloot PM, Lloyd CM, Filatov MV. Calcium homeostasis and signaling in yeast cells and cardiac myocytes. FEMS Yeast Res. 2009:9:1137-47.

8. Colinet AS, Thines L, Deschamps A, Flémal G, Demaegd D, Morsomme P. Acidic and uncharged polar residues in the consensus motifs of the yeast $\mathrm{Ca}^{2+}$ transporter Gdt1p are required for calcium transport. Cell Microbiol. 2017:19(7). https://doi.org/https://doi.org/10.1111/cmi. 12729.

9. Zhao $Y$, Yan H, Happeck R, Peiter-Volk T, Xu H, Zhang Y, Peiter E, van Oostende TC, Whiteway M, Jiang L. The plasma membrane protein Rch1 is a negative regulator of cytosolic calcium homeostasis and positively regulated by the calcium/calcineurin signaling pathway in budding yeast. Eur J Cell Biol. 2016;95:164-74.

10. Cyert MS, Philpott CC. Regulation of cation balance in Saccharomyces cerevisiae. Genetics. 2013;193:677-713.

11. Zhao Y, Du J, Zhao G, Jiang L. Activation of calcineurin is mainly responsible for the calcium sensitivity of gene deletion mutations in the genome of budding yeast. Genomics. 2013;101:49-56.

12. Dadar M, Tiwari R, Karthik K, Chakraborty S, Shahali Y, Dhama K. Candida albicans - Biology, molecular characterization, pathogenicity, and advances in diagnosis and control - An update. Microb Pathog. 2018;117:128-38.

13. Yu SJ, Chang YL, Chen YL. Calcineurin signaling: lessons from Candida species. FEMS Yeast Res. 2015;15(4). https://doi.org/https://doi.org/10.1093/ femsyr/fov016

14. Chauhan N, Latge JP, Calderone R. Signalling and oxidant adaptation in Candida albicans and Aspergillus fumigatus. Nat Rev Microbiol. 2006:4:435-44

15. Jiang L, Xu D, Hameed A, Fang T. Bakr Ahmad Fazili a, Asghar F. The plasma membrane protein Rch1 and the Golgi/ER calcium pump Pmr1 have an additive effect on filamentation in Candida albicans. Fungal Genet Biol. 2018;115:1-8.

16. Jiang L, Alber J, Wang J, Du W, Li X, Geyer J. The Candida albicans plasma membrane protein Rch1p a member of the vertebrate SLC10 carrier family, is a novel regulator of cytosolic $\mathrm{Ca}^{2+}$ homoeostasis. Biochem J. 2012:444:497-502.

17. Liu S, Hou Y, Liu W, Lu C, Wang W, Sun S. Components of the calciumcalcineurin signaling pathway in fungal cells and their potential as antifungal targets. Eukaryot Cell. 2015;14:324-34

18. Wang Y, Wang J, Cheng J, Xu D, Jiang L. Genetic interactions between the Golgi $\mathrm{Ca}^{2+} / \mathrm{H}^{+}$exchanger Gdt1 and the plasma membrane calcium channel Cch1/Mid1 in the regulation of calcium homeostasis, stress response and virulence in Candida albicans. FEMS Yeast Res. 2015;15:(7). https://doi.org/ https://doi.org/10.1093/femsyr/fov069.

19. Xu D, Cheng J, Cao C, Wang L, Jiang L. Genetic interactions between Rch and the high-affinity calcium influx system Cch1/Mid1/Ecm7 in the regulation of calcium homeostasis, drug tolerance, hyphal development 
and virulence in Candida albicans. FEMS Yeast Res. 2015;15:(7). https://doi. org/https://doi.org/10.1093/femsyr/fov079.

20. Alber J, Jiang L, Geyer J. CaRch1p does not functionally interact with the high-affinity $\mathrm{Ca}^{2+}$ influx system (HACS) of Candida albicans. Yeast. 2013;30:449-57.

21. Reedy JL, Filler SG, Heitman J. Elucidating the Candida albicans calcineurin signaling cascade controlling stress response and virulence. Fungal Genet Biol. 2010;47:107-16.

22. Li X, Hou Y, Yue L, Liu S, Du J, Sun S. Potential targets for antifungal drug discovery based on growth and virulence in Candida albicans. Antimicrob Agents Chemother. 2015;59:5885-91.

23. Thewes S. Calcineurin-Crzl signaling in lower eukaryotes. Eukaryot Cell. 2014;13:694-705.

24. Gow NA, van de Veerdonk FL, Brown AJ, Netea MG. Candida albicans morphogenesis and host defence: discriminating invasion from colonization. Nat Rev Microbiol. 2011;10:112-22.

25. Sanglard D, Ischer F, Marchetti O, Entenza J, Bille J. Calcineurin a of Candida albicans: involvement in antifungal tolerance, cell morphogenesis and virulence. Mol Microbiol. 2003:48:959-76.

26. Blankenship JR, Wormley FL, Boyce MK, Schell WA, Filler SG, Perfect JR, Heitman J. Calcineurin is essential for Candida albicans survival in serum and virulence. Eukaryot Cell. 2003;2:422-30.

27. Cruz MC, Goldstein AL, Blankenship JR, del Poeta M, Davis D, Cardenas ME, Perfect JR, McCusker JH, Heitman J. Calcineurin is essential for survival during membrane stress in Candida albicans. EMBO J. 2002;21:546-59.

28. Karababa M, Valentino E, Pardini G, Coste AT, Bille J, Sanglard D. CRZ1, a target of the calcineurin pathway in Candida albicans. Mol Microbiol. 2006;59:1429-51

29. Onyewu C, FLJ W, Perfect JR, Heitman J. The calcineurin target, Crz1, functions in azole tolerance but is not required for virulence of Candida albicans. Infect Immun. 2004;72:7330-3.

30. Xu H, Whiteway M, Jiang L. The tricarboxylic acid cycle, cell wall integrity pathway, cytokinesis and intracellular pH homeostasis are involved in the sensitivity of Candida albicans cells to high levels of extracellular calcium. Genomics. 2018;pii: S0888-7543(18)30307-0. https://doi.org/https://doi.org/ 10.1016/j.ygeno. 2018.08.001.

31. Chen Y, Mallick J, Magnas A, Sun Y, Choudhury BI, Cote P, Yan L, Ni TJ, Li Y, Zhang D, Rodríguez-Ortiz R, Lv QZ, Jiang YY, Whiteway M. Chemogenomic profiling of the fungal pathogen Candida albicans. Antimicrob Agents Chemother. 2018;62:pii: e02365-17. https://doi.org/https://doi.org/10.1128/ AAC.02365-17.

32. Roemer T, Jiang B, Davison J, Ketela T, Veillette K, Breton A, Tandia F, Linteau A, Sillaots S, Marta C, Martel N, Veronneau S, Lemieux S, Kauffman S, Becker J, Storms R, Boone C, Bussey H. Large-scale essential gene identification in Candida albicans and applications to antifungal drug discovery. Mol Microbiol. 2003;50:167-81.

33. Mille C, Janbon G, Delplace F, Ibata-Ombetta S, Gaillardin C, Strecker G, Jouault T, Trinel PA, Poulain D. Inactivation of CaMIT1 inhibits Candida albicans phospholipomannan beta-mannosylation, reduces virulence, and alters cell wall protein beta-mannosylation. J Biol Chem. 2004;279:47952-60.

34. Zhao W, He X, Hoadley KA, Parker JS, Hayes DN, Perou CM. Comparison of RNA-Seq by poly (A) capture, ribosomal RNA depletion, and DNA microarray for expression profiling. BMC Genomics. 2014;15:419.

35. Kogenaru S, Qing Y, Guo Y, Wang N. RNA-seq and microarray complement each other in transcriptome profiling. BMC Genomics. 2012;13:629.

36. Wang Z, Gerstein M, Snyder M. RNA-Seq: a revolutionary tool for transcriptomics. Nat Rev Genet. 2009;10:57-63.

37. Nagalakshmi U, Wang Z, Waern K, Shou C, Raha D, Gerstein M, Snyder M. The transcriptional landscape of the yeast genome defined by RNA sequencing. Science. 2008;320:1344-9.

38. Sun Y, Gadoury C, Hirakawa MP, Bennett RJ, Harcus D, Marcil A, Whiteway M. Deletion of a Yci1 domain protein of Candida albicans allows homothallic mating in MTL heterozygous cells. MBio. 2016;7:(2). https://doi: https://doi.org/10.1128/mBio.00465-16

39. Vyas VK, Barrasa IM, Fink GR. A Candida albicans CRISPR system permits genetic engineering of essential genes and gene families. Sci Adv. 2015;1: (3). https://doi:https://doi.org/10.1126/sciadv.1500248

40. Brand A, Lee K, Veses V, Gow NA. Calcium homeostasis is required for contact-dependent helical and sinusoidal tip growth in Candida albicans hyphae. Mol Microbiol. 2009;71:1155-64.
41. Asghar F, Yan H, Jiang L. The putative transcription factor CaMaf1 controls the sensitivity to lithium and rapamycin and represses RNA polymerase III transcription in Candida albicans. FEMS Yeast Res. 2018;18:(6). https://doi. org/https://doi.org/10.1093/femsyr/foy068.

42. Zhao Y, Feng J, Li J, Jiang L. Mithochondrial type $2 \mathrm{C}$ protein phosphatases CaPtc5p, CaPtc6p, and CaPtc7p play vital roles in cellular responses to antifungal drugs and cadmium in Candida albicans. FEMS Yeast Res. 2012;12:897-906.

43. Yu L, Zhao J, Feng J, Fang J, Feng C, Jiang Y, Cao Y, Jiang L. Candida albicans CaPTC6 is a functional homolog for Saccharomycese cerevisiae SCPTC6 and encodes a type 2C protein phosphatase. Yeast. 2010;27:197-206.

44. Jiang L, Whiteway M, Shen $\mathrm{SH}$. A novel type $2 \mathrm{C}$ protein phosphatase from the human fungal pathogen. Candida albicans FEBS Lett. 2001;509:42-144.

45. Jiang L, Wang J, Asghar F, Snyder N, Cunningham KW. CaGdt1 plays a compensatory role for the calcium pump CaPmr1 in the regulation of calcium signaling and cell wall integrity signaling in Candida albicans. Cell Commun Signal. 2018a; 16:33.

46. Yan $\mathrm{H}$, Zhao Y, Jiang L. The putative transcription factor CaRtg3 is involved in tolerance to cations and antifungal drugs as well as serum-induced filamentation in Candida albicans. FEMS Yeast Res. 2014;14:614-23.

47. Zhao Y, Du J, Xiong B, Xu H, Jiang L. ESCRT components regulate the expression of the ER/Golgi calcium pump gene PMR1 through the Rim101/ Nrg1 pathway in budding yeast. J Mol Cell Biol. 2013;5:336-44.

48. Yoshimoto H, Saltsman K, Gasch AP, Li HX, Ogawa N, Botstein D, Brown PO, Cyert MS. Genome-wide analysis of gene expression regulated by the calcineurin/Crz1p signaling pathway in Saccharomyces cerevisiae. J Biol Chem. 2002:277:31079-88.

49. Kim S, Hu J, Oh Y, Park J, Choi J, Lee YH, Dean RA, Mitchell TK. Combining ChIP-chip and expression profiling to model the MoCRZ1 mediated circuit for $\mathrm{Ca} /$ calcineurin signaling in the rice blast fungus. PLoS Pathog. 2010;6:(5). https://doi.org/https://doi.org/10.1371/ journal.ppat.1000909.

50. Choi J, Kim Y, Kim S, Park J, Lee YH. MoCRZ1, a gene encoding a calcineurin-responsive transcription factor, regulates fungal growth and pathogenicity of Magnaporthe oryzae. Fungal Genet Biol. 2009;46:243-54.

51. Miranda I, Rocha R, Santos MC, Mateus DD, Moura GR, Carreto L, Santos MA. A genetic code alteration is a phenotype diversity generator in the human pathogen Candida albicans. PLoS One. 2007;2:(10). https://doi:https://doi. org/10.1371/journal.pone.0000996

52. Schena M, Shalon D, Davis RW, Brown PO. Quantitative monitoring of gene expression patterns with a complementary DNA microarray. Science. 1995;270:467-70.

53. Mortazavi A, Williams BA, McCue K, Schaeffer L, Wold B. Mapping and quantifying mammalian transcriptomes by RNA-Seq. Nat Methods. 2008;5:621-8.

54. Fu C, Donadio N, Cardenas ME, Heitman J. Dissecting the roles of the calcineurin pathway in unisexual reproduction, stress responses, and virulence in Cryptococcus deneoformans. Genetics. 2018:208:639-53.

55. Fox DS, Heitman J. Calcineurin-binding protein Cbp1 directs the specificity of calcineurin-dependent hyphal elongation during mating in Cryptococcus neoformans. Eukaryot Cell. 2005;4:1526-38.

56. Kraus PR, Heitman J. Coping with stress: calmodulin and calcineurin in model and pathogenic fungi. Biochem Biophys Res Commun. 2003:311:1151-7.

57. Soriani FM, Mala I, da Silva Ferreira ME, Savoldi M, von Zeska Kress MR, de Souza Goldman MH, Loss O, Bignell E, Goldman GH. Functional characterization of the Aspergillus fumigatus CRZ1 homologue. CrzA Mol Microbiol. 2008;67:1274-91.

58. Kingsbury TJ, Cunningham KW. A conserved family of calcineurin regulators. Genes Dev. 2000;14:1595-604.

\section{Publisher's Note}

Springer Nature remains neutral with regard to jurisdictional claims in published maps and institutional affiliations. 